\title{
An Evaluation of Microbial Flora, Alkaline Phosphatase and IL-8 Levels in GCF of Orthodontic Patients with Self-Ligating and Conventional Brackets
}

\author{
Essam Abdelalim Nassar (D) \\ Naif Nasser Almasoud' \\ Maher Sulaiman Al- \\ Qurashi ${ }^{2}$ \\ Ahmed A Alsulaiman (D) \\ Khalid Sadiaq Hassan ${ }^{3}$ \\ 'Department of Preventive Dental \\ Sciences, College of Dentistry, Imam \\ Abdulrahman Bin Faisal University, \\ Dammam, Saudi Arabia; ${ }^{2}$ Department of \\ Microbiology, College of Medicine, Imam \\ Abdulrahman Bin Faisal University, \\ Dammam, KSA, Saudi Arabia; \\ ${ }^{3}$ Department of oral Medicine and \\ Periodontology, College of Dentistry, Al- \\ Azhar University, Assiut, Egypt
}

Purpose: To compare the microbial flora, alkaline phosphatase (ALP) and interleukin-8 (IL8) levels during orthodontic treatment with self-ligating brackets versus conventional brackets.

Patients and methods: Forty patients were included in this study and distributed equally between two groups. One group received conventional edgewise brackets while the other group received DAMON self-ligating brackets. Periodontal parameters were recorded and biochemical and microbial analyses were conducted before bonding (baseline), and 14, 30, and 45 days after bonding. Paired $t$-test was performed to estimate the intergroup differences from the baseline at the various time points; unpaired $t$-test was used for intragroup comparisons.

Results: A significantly higher prevalence of gram-negative as well as gram-positive microorganisms was found in the group with conventional brackets than that with selfligating brackets. Alkaline phosphatase (ALP) activity and interleukin-8 (IL-8) levels were significantly higher in the gingival crevicular fluid of the group with conventional brackets than that with self-ligating brackets.

Conclusion: The conventional bracket-ligature technique exhibited an increase in IL- 8 levels, ALP activity, and microbial colonization compared with that of the self-ligature technique. Accordingly, self-ligating brackets are recommended in orthodontic patients to improve periodontal health and minimize damage outcomes of periodontal tissue interventions.

Keywords: dentistry, orthodontics, alkaline phosphatase, jnterlukin- 8 , orthodontic tooth movement, periodontal parameters

\section{Introduction}

Tooth movement during orthodontic treatment occurs due to tissue remodeling driven by the host inflammatory response. Orthodontic tooth movement is accompanied by the release of numerous regulatory molecules, such as cytokines. ${ }^{1}$ Cytokines are classified as proinflammatory and anti-inflammatory and considered as key mediators of tissue remodeling. Elevated concentrations of cytokines, such as interleukin-6 (IL-6), IL-1b, IL-8, tumor necrosis factor-a (TNF-a), epidermal growth factor, and b2 microglobulin, are observed during orthodontic tooth movement. ${ }^{1-3}$ The release of IL- 6 and IL- 8 in the periodontal ligament (PDL) in
Correspondence: Essam Abdelalim Nassar

Department of Preventive Dental

Sciences, College of Dentistry, Imam

Abdulrahman Bin Faisal University, Saudi

Arabia

Tel +966542941098

Email e_abdelalim@yahoo.com 
turn triggers a host immune response distinguished by the chemoattraction of immune cells to clear cellular debris and assist in the re-establishment of the periodontal architecture in the initial phase and tooth movement in an advanced stage. ${ }^{4,5}$ Furthermore, an upregulation of IL-17 and IL-23 follows the application of force at the compression sites. ${ }^{6}$ A similar finding has been demonstrated for prostaglandin E and IL-1b. ${ }^{7}$

The biochemical mediators expressed during orthodontic tooth movement can be isolated from gingival crevicular fluid (GCF). GCF is a distinctive biological exudate that is considered as an appropriate medium for noninvasive assessment of biochemical mediators and their associated biological events throughout the observation, with rational sensitivity. ${ }^{8,9}$

Alkaline phosphatase (ALP) is a key enzyme that regulates bone mineralization by releasing an organic phosphate that precipitates the calcium-phosphate complex into the osteoid matrix. ${ }^{10}$ It can also inhibit the mineralization by hydrolyzing inorganic pyrophosphate, which prevents hydroxyapatite crystal formation. ${ }^{11,12}$ Fibroblasts of PDL and osteoblasts of the alveolar bone are capable of producing ALP. ${ }^{13}$

Ligation procedures can influence the surrounding microbiota. Forsberg et al and Turkkahraman et al compared the microbes between brackets ligated with an elastomeric ring and those with a steel wire; they found a higher number of microorganisms in the plaques of teeth in the former. ${ }^{14,15}$ Their results concluded that elastomeric rings should be avoided in patients with poor oral hygiene, as it would certainly increase microbial colonization on tooth surfaces adjacent to the brackets. Selfligating brackets (SLB) are orthodontic brackets that require neither elastomeric ligation methods nor stainless steel wire ligatures to hold the orthodontic archwires in place. Currently, different shapes and designs of SLB are available in the market and these can be classified into passive or active types depending on whether they generate forces on the archwires, or not. Numerous advantages are commercially attributed to the SLB and include the reduction in friction between the brackets, quicker alignment and lowering gaps, superior expansion of the arch with reduced incisor proclination, minimal extraction to gain space and relieve crowding, shorter appointment times, shorter overall-treatment period, and a better oral hygiene owing to the reduced complexity of the brackets with fewer retentive sites for microbial colonization. ${ }^{16-19}$ The purpose of this research was to estimate the periodontal parameters, IL-8 level and ALP activity in GCF, and assess the microbial flora during orthodontic tooth movement in patients treated with either SLB or conventional ligatures.

\section{Materials and Methods Patient Information and Orthodontic Procedures}

This prospective clinical study included 40 patients (18 males and 22 females) selected from the outpatient clinics to receive orthodontic treatment at Orthodontic Division, College of Dentistry, Imam Abdulrahman Bin Faisal University. The patients were aged $12-18$ years and divided into two groups by random selection. Twenty patients ( 8 males and 12 females; mean age $15.4 \pm 1.8$ years) treated with edgewise brackets (Orthos CM, Ormco, Glendora, CA, USA) and elastic ligature technique (Dentalastics ${ }^{\circledR}$, Dentaurum, Ispringen, Germany) constituted the control group (group 1), while 20 patients (10 males and 10 females; mean age $15.6 \pm 2.1$ years) who received SLB treatment (DAMON Q, Ormco) constituted group 2. A flow chart of the experimental design is illustrated in Figure 1.

Sample size calculation was based on the post-hoc power analysis using G Power (version 3.1.5; Franz Faul, University Kiel, Germany) with effect size $\mathrm{d}=1$, power set at 0.80 , and alpha $=0.05$ (two-tailed). The total sample size required was at least 18 participants per group. The primary outcome was defined as the amount of change in mean colony forming unit. ${ }^{14,20,21}$ All selected patients met the following inclusion criteria: good health, no history of systemic diseases, not under medications such as antibiotics or anti-inflammatory drugs in the past 6 months prior to onset of this study, absence of restorations, no reported oral habits detrimental to health, including smoking and/or missing teeth due to dental caries. An orthodontic treatment plan begins with alignment and leveling as the first stage in upper and lower arches. A 0.014-in copper-nickel-titanium archwire was used for the initial leveling. During the study period, no additional materials, such as chains, coil springs, or Figure-8 ligatures, which could have adversely affected oral hygiene, were used.

Prior to the orthodontic treatment, written informed consent was obtained from all patients and parents of patients under 18 years. The protocol was approved from the ethical committee of Imam Abdulrahman Bin Faisal 


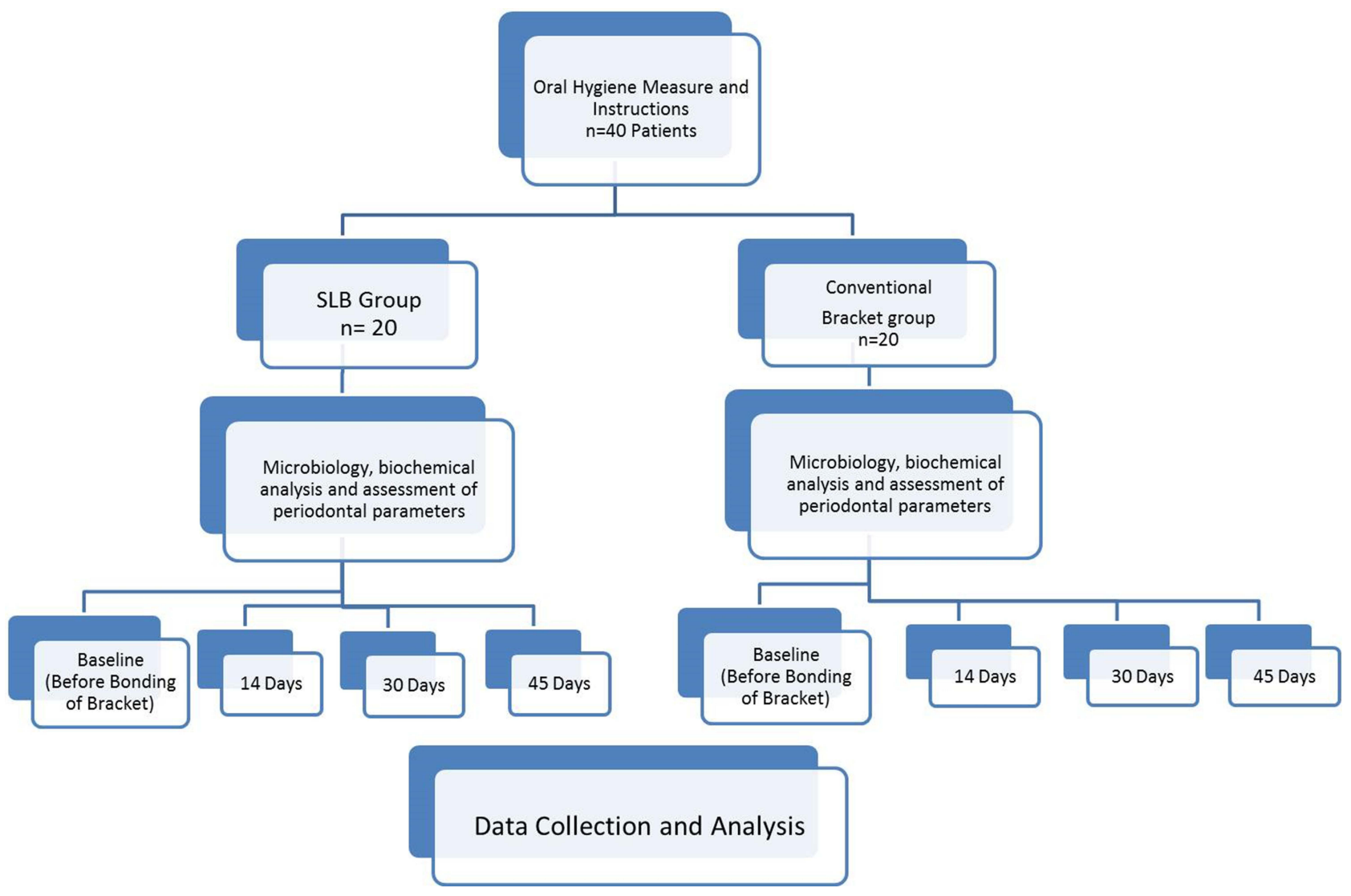

Figure I Flow chart of the experiment design.

University and the study was conducted in accordance with the Declaration of Helsinki.

Supra- and subgingival scaling, root planning, and oral hygiene procedures were performed for each patient via manual and ultrasonic instrumentation. All the patients were re-evaluated after four weeks. Patients are required to achieve a good oral hygiene prior to proceeding with the placement of brackets. Oral hygiene was assessed visually and verbally. A good plaque control was visually assessed by the absence of plaque accumulation on teeth and marginal gingiva, and the absence of marginal inflammation. Verbal assessment involved the patient's response in negative to the query if there was any bleeding in the gums after brushing. Orthodontic treatment was performed only if both criteria were met. Additional reinforcement of oral hygiene was implemented in the regular checkups every 4-5 weeks.

Biochemical and microbial analysis and periodontal parameters were assessed before bonding (baseline), and 14,30 , and 45 days after bonding. Periodontal measurements were recorded at six sites per tooth, on the selected teeth (upper left canine, upper right first molar, upper left first premolar, lower right canine, lower left first molar, and lower left first premolars numbered 11, 3, 12, 27, 19, and 21 respectively, according to the universal numbering system).

Measurements were recorded by a single examiner blinded for the treatment provided by the orthodontist (single-blinded). A certified periodontist performed the measurements for plaque index (PI), gingival index (GI), and bleeding on probing (BOP). The same examiner calibrated and evaluated the probing pocket depth (PPD). Calibration was performed with a Michigan "O" periodontal probe with Williams marking (Hu-Friedy, Chicago, IL, USA). Duplicate examinations at an interval of one week were performed on five patients. Statistical analysis of calibration results was accomplished using the kappa test. The intra-examiner value for kappa was 0.78 , indicating substantial reliability. The values of PI, GI, PPD, and BOP within $15 \mathrm{~s}$ after probing with a $20 \mathrm{~g}$ controlled-force probe used for the periodontal evaluation. ${ }^{22,23}$

\section{Microbiological Analysis}

Subgingival plaque and GCF were collected individually with a standardized sterile paper strip \#30. After isolation of the teeth with cotton rolls and air drying, a strip was 
Table I Periodontal Clinical Parameters in Conventional Bracket Group and SLB Group

\begin{tabular}{|l|c|c|c|c|}
\hline \multicolumn{2}{|l|}{ Variables } & $\begin{array}{c}\text { Conventional Bracket } \\
\mathbf{n = 2 0}\end{array}$ & SLB $\mathbf{n = 2 0}$ & p-value \\
\hline GI & Mean \pm SD & $1.9 \pm 2.4$ & $1.2 \pm 2.3$ & $0.003^{*}$ \\
$\%$ sites with PI & Mean \pm SD & $57.5 \pm 17.6$ & $43.5 \pm 20.1$ & $0.00 I^{*}$ \\
\% sites with BOP & Mean \pm SD & $46.4 \pm 21.3$ & $43.5 \pm 20.1$ & $0.00 I^{*}$ \\
PPD (mm) & Mean \pm SD & $4.6 \pm 0.4$ & $2.4 \pm 0.3$ & $0.003^{*}$ \\
\hline
\end{tabular}

Note: *Significant at p-value $<0.05$.

placed into the gingival crevices at mesial and distal positions of canine, molar, and premolars at $1 \mathrm{~mm}$ depth for 10 s. Subsequently, each microbial sample was placed immediately into a vial containing $0.5 \mathrm{~mL}$ of the reduced transport fluid, thioglycolate broth (L-cysteine 0.5 , sodium chloride 2.5, glucose 5.5, yeast extract 5.0, pancreatic digest casein 15.0, and sodium thioglycolate 0.5 [units in $\mathrm{g} / \mathrm{L}$ ]). ${ }^{24}$ The vials were filled with nitrogen and transported within 40 min, to the Microbiology Diagnostic Laboratory, College of Medicine, Imam Abdulrahman Bin Faisal University.

\section{Biochemical Analysis}

GCF was sampled discretely from the mesial and distal gingival crevices for each canine molar, and premolars using precut paper filter strips (Periopaper gingival fluid collection strips, ProFlow Inc., North Haven, CT, USA). Strips were inserted with $5 \mathrm{~s}$ intervals into the gingival crevice until minor resistance was felt and retained in situ for $60 \mathrm{~s}$.

GCF volumes were calculated with an electronic instrument (Periotron 8000, Ora Flow, CA, USA) that was standardized by as per manufacturer's instructions. A software program (MLCONVERT.EXE ver. 2.52, Ora Flow, NY, USA) was used to convert the quantities to microliters. ${ }^{25}$ After the collection of GCF, the paper strips were incubated in $200 \mu \mathrm{L}$ of saline for $15 \mathrm{~min}$, the strips discarded and the remaining tubes with the fluids were transported to the College of Medicine biochemistry lab, and stored at $-70^{\circ} \mathrm{C}$ until biochemical analysis. ${ }^{26}$

\section{Statistical Analysis}

Statistical package for the social sciences version 18 (SPSS Inc., Chicago, IL, USA) was used to analyze the data. Descriptive statistics were employed for all variables. The baseline values evaluated for the homogeneity of the groups (excluding the relative values) were analyzed by unpaired $t$-test. Paired $t$-test was used to estimate the differences from the baseline to the various monitoring periods in both groups. The unpaired $t$-test was used for intragroup comparisons. The level of significance was established at $P \leq 0.05$.

\section{Results}

The clinical data for the GI, PI \%, BOP \%, and PPD are shown in Table 1. Significant differences were observed in the clinical parameters between the SLB and conventional brackets group $(P<0.05)$.

Table 2 compares the prevalence of different species of subgingival bacteria between the two groups. A significantly higher prevalence of gram-negative microorganisms was found in the conventional brackets group than in the SLB group ( $41.6 \%$ and $25 \%$, respectively, $P=0.001)$. Likewise, the prevalence of gram-positive organisms was significantly higher in the conventional bracket group compared with the SLB group (33.3\% and $26.7 \%$, respectively, $P=0.02$ ).

ALP levels in the two groups at the time intervals from baseline to 45 days are shown in Table 3 and Figure 2. There was no significant change in the ALP levels at the baseline between the groups. However, following bracket bonding and insertion of initial archwire through the different periods of treatment, the ALP levels significantly differed between the two groups $(P<0.05)$. ALP levels in the GCF were elevated with an increase in the duration of treatment session and the duration of orthodontic tooth movements.

Table 4 and Figure 3 illustrate the levels of IL- 8 in GCF in the two groups at the different time intervals. There was no significant difference in the IL-8 levels at the baseline between the two groups. However, these differences were highly significant at 14, 30, and 45 days after bracket bonding and start of treatment plan $(P<0.05)$.

\section{Discussion}

Orthodontic treatment generally involves the use of biomechanical forces on teeth. The frequency, duration, and force magnitudes of orthodontic treatments influence the adjacent tissue reaction and bone remodeling. The interaction between bone deposition and resorption results in the 
Table 2 Prevalence of Subgingival Bacteria in Conventional Bracket Group and SLB Group

\begin{tabular}{|l|c|c|c|}
\hline Variable & Conventional Bracket & SLB & p-value \\
\hline Bifidobacterium species (GPR) & 0 & $4(13.3)$ & $0.00 I^{*}$ \\
Clostridium ramosum (GPR) & 0 & $4(13.3)$ & $0.005^{*}$ \\
Peptococcus asaccharolyticus (GPC) & 0 & $4(13.3)$ & $0.005^{*}$ \\
Prevotella melaninogenicus (GNR) & $11(36.6)$ & 0 & $0.00 I^{*}$ \\
Prevotella oralis (GNR) & $10(33.3)$ & 0 & $0.00 I^{*}$ \\
Fusobacterium mortiferum (GNB) & $4(13.3)$ & $4(13.3)$ & 1 \\
Peptostreptococcus group (GPC) & $8(26.6)$ & $11(36.6)$ & 0.28 \\
Eubacterium limosum (GPR) & $4(13.3)$ & 0 & $0.00 I^{*}$ \\
Veilonella parvula (GNC) & $4(13.3)$ & 0 & 1 \\
Actinomyces meyeri (GPR) & $4(13.3)$ & 0 & $0.005^{*}$ \\
Actinomyces odontylicus (GPR) & $4(13.3)$ & 0 & $0.004^{*}$ \\
Porphyromonas asaccharolytica (GNB) & $2(6.6)$ & $15(25)$ & 0.38 \\
Gram negative organism & $25(41.6)$ & $16(26.7)$ & $0.00 I^{*}$ \\
Gram positive organism & $20(33.3)$ & $0.02^{*}$ \\
\hline
\end{tabular}

Note: *Significant at $\mathrm{p}$-value $<0.05$.

Table 3 ALP Activity $(\mu L)$ in GCF in Conventional Bracket Group and SLB Group

\begin{tabular}{|c|c|c|c|c|c|}
\hline & & & $\begin{array}{c}\text { Conventional } \\
\text { Bracket }\end{array}$ & SLB & p-value \\
\hline \multicolumn{2}{|c|}{ ALK $(\mu \mathrm{L})$} & Mean $\pm S D$ & $100.15 \pm 23.4$ & $88.92 \pm 12.32$ & $0.019 *$ \\
\hline \multirow[t]{4}{*}{ Time } & 0 Day & Mean \pm SD & $63.71 \pm 7.24$ & $64.18 \pm 4.38$ & 0.013 \\
\hline & I4 Days & Mean $\pm S D$ & $104.45 \pm 3.99$ & $86.57 \pm 6.63$ & $0.000 *$ \\
\hline & 30 Days & Mean $\pm S D$ & II $2.52 \pm 11.79$ & $95.04 \pm 7.27$ & $0.003 *$ \\
\hline & 45 Days & Mean $\pm S D$ & $119.92 \pm 7.80$ & $100.88 \pm 5.21$ & $0.000 *$ \\
\hline
\end{tabular}

Note: *Significant at $p$-value $<0.05$.

discharge of different biochemical or cellular mediators. ${ }^{27}$ The recognition of remodeling patterns in the PDL during orthodontic treatment via assessment of the biochemical mediators may be clinically beneficial due to their key roles in tooth movement and tissue damage. ${ }^{6,28}$

The results of this study clearly demonstrate that IL-8 production in the conventional brackets group was greater than in the SLB group at the different time intervals probed. IL-8 is crucial in controlling alveolar bone resorption during tooth movement and is responsible for recruiting and activating neutrophils during inflammation. ${ }^{3}$ It is also suggested that IL-8 plays a multifactorial role in the pathogenesis of the periodontal disease. King et al reported that marked tissue destruction at the tension sites at various stages of tooth movements leads to a rise in the biochemical activity of GCF mediators at the tension site. ${ }^{11}$ Our findings matched those of Başaran et al, ${ }^{29}$ who demonstrated changes in the IL-8 levels with the application of orthodontic forces. Interestingly, the level of IL-8 was higher in patients treated with aligners compared to those treated by labial fixed appliances. ${ }^{30}$
The relation between the orthodontic mechanical forces and collagen turnover is important in tooth movement. In this respect, previous studies have demonstrated that orthodontic forces increased DNA synthesis, collagen synthesis, and ALP activity. ${ }^{31,32}$ In the present study, the levels of ALP were significantly higher 14, 30, and 45 days after the application of orthodontic forces in both the groups. This is in accordance with several studies who reported an increased ALP level at 1, 2, and 3 weeks after force application. ${ }^{33,34}$ Additionally, a significant increase in the GCF ALP concentration was observed with an increased duration of orthodontic tooth movement, thus implying a direct correlation between ALP and the bone remodeling process. $^{35}$

Corrective orthodontics produce clinical periodontal variations with respect to biofilm accumulation, gingival bleeding and periodontal pathogens. ${ }^{36}$ In this study, we compared the effects of SLB and conventional brackets ligated with elastomeric rings despite the fact that elastomeric ligatures are considered as biohostile materials. 


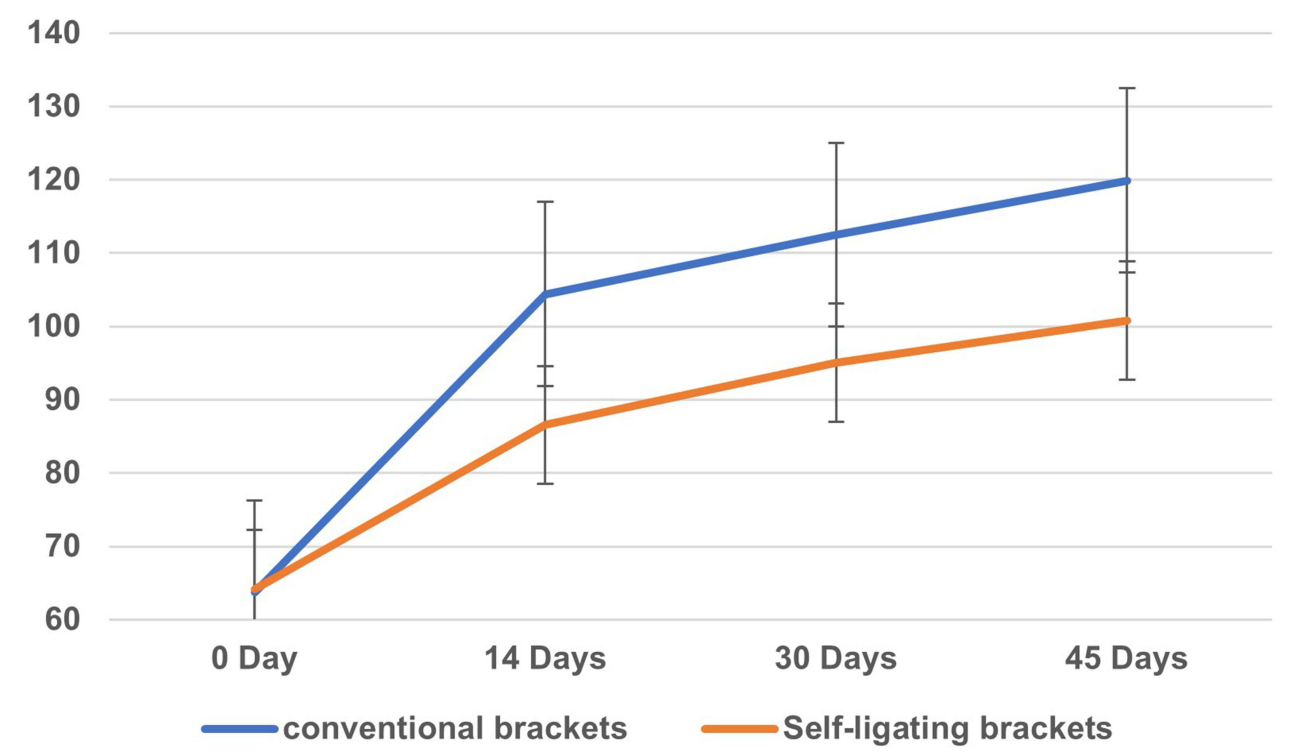

Figure 2 ALP levels (UL) in GCF for conventional bracket group and SLB group at different treatment periods.

Elastomeric rings are convenient to use and represent the choice for both patients and the orthodontists. In contrast, the stainless steel ligatures are time overwhelming and infrequently used in clinical practice. The findings of the present study prove that there is an increase in the number of gram-negative and gram-positive microorganisms with the use of conventional brackets ligated with elastomeric rings as against when SLBs are used. These findings are in agreement with Hassan et al who concluded that the archwire ligature technique initiated microbial colonization and aspartate aminotransferase activity more than the selfligature technique. ${ }^{26}$ Jingh et al reported that the percentage of Porphyromonas gingivalis is lower in SLBs than in conventional brackets. ${ }^{37}$ Additionally, Pejda et al found statistically greater incidence of Aggregatibacter actinomycetemcomitans in patients with conventional brackets ligated with steel ligatures than in those with SLBs. ${ }^{21}$ Instead, our findings are in disagreement with Sukontapatipark et al who highlighted that the bacterial colonization was not affected by the ligation pattern. ${ }^{38}$
This disagreement can be attributable to the following reasons: variation in observation timepoints, microbiological analysis, the population studied, statistical analyses, and differences in manufacturer of the brackets.

The marked increase in bacterial colonization and subsequent periodontal pathogenesis in the conventional brackets group compared to the SLB group corresponded to significant differences in the clinical parameters (GI, PI, BOP, and PPD) between the groups $(P<0.05)$. These findings are in contrast to other investigations that declared no differences in the periodontal status in patients who were treated with either SLB or conventional brackets. ${ }^{39-}$ 42 This difference can be explained by the following aspects: variation in baseline confounders, observation timepoints, methodological design, the population studied, units of assessment, statistical analyses, and differences in manufacturer of the brackets. Additionally, meta-analyses described in the literature have expressed the issue of low quality of evidence in studies that evaluated the difference of periodontal status in patients who were treated with

Table 4 IL-8 GCF Levels in Conventional Bracket Group and SLB Group

\begin{tabular}{|l|c|c|c|c|c|}
\hline \multicolumn{2}{|l|}{} & Conventional Bracket & SLB & P value \\
\hline TL-8 & & Mean \pm SD & $11.87 \pm 9.04$ & $2.46 \pm 1.72$ & $0.01 *$ \\
\hline \multirow{2}{*}{ Time } & O Day & Mean \pm SD & $0.88 \pm 0.31$ & $0.64 \pm 0.31$ & 0.138 \\
& I4 Days & Mean \pm SD & $9.17 \pm 2.24$ & $2.60 \pm 1.13$ & $<0.000 I^{*}$ \\
& 30 Days & Mean \pm SD & $19.1 \pm 8.08$ & $2.8 \pm 1.63$ & $<0.000 I^{*}$ \\
& 45 Days & Mean \pm SD & $18.32 \pm 6.14$ & $3.8 \pm 1.73$ & $<0.000 I^{*}$ \\
\hline
\end{tabular}

Note: *Significant at p-value $<0.05$. 


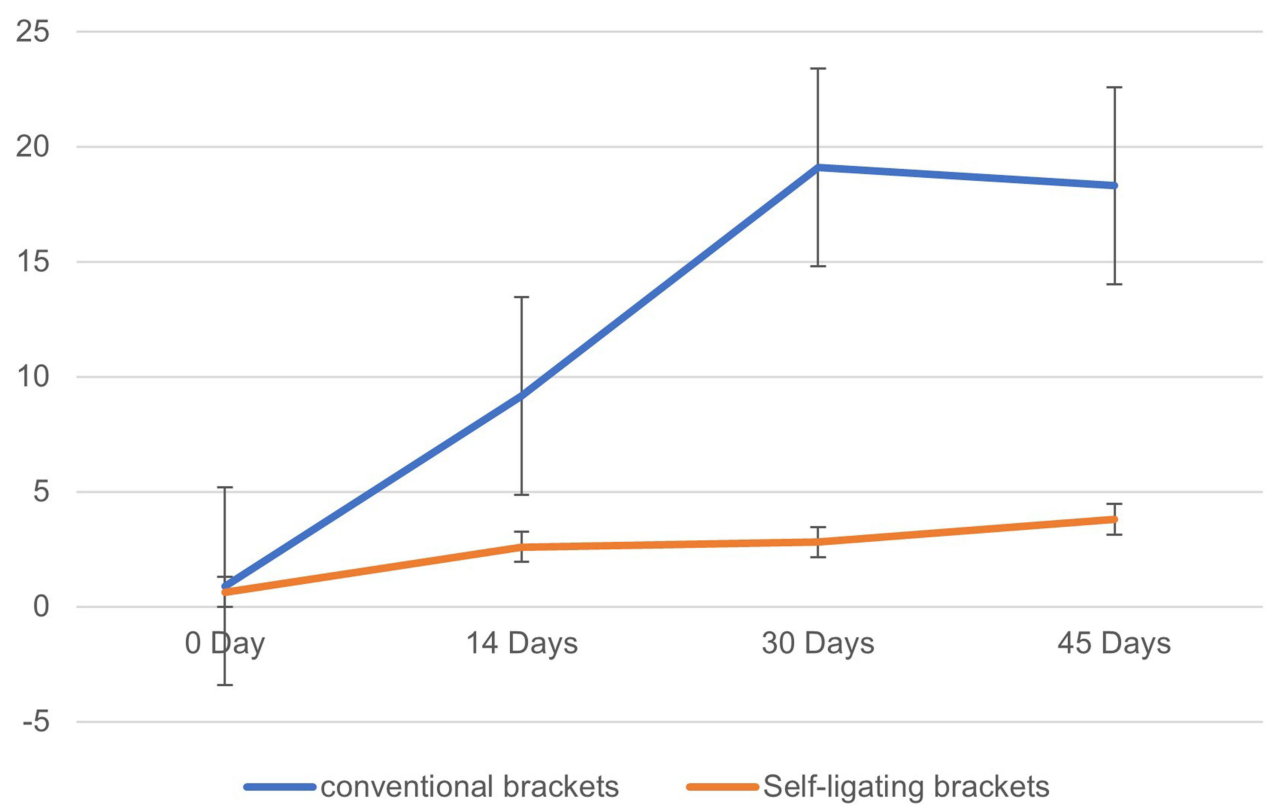

Figure 3 Comparison between IL-8 levels in GCF for conventional bracket group and SLB group.

SLB or conventional brackets. Their results cannot be generalized yet, as the integrated Randomized Clinical Trials present high heterogeneity and a risk of high bias.

Moreover, a significant correlation between periodontal conditions and GCF biomarkers of inflammation cannot be omitted. Nunes et al found that most of the correlations between both PI \%, and BOP \% with the GCF cytokine levels were not significant. ${ }^{43}$ However, the high variability and small sample size in their study have to be considered when interpreting this evidence. Further studies assessing this correlation would be of merit.

Light continuous forces are considered more preferable for tooth movements. They allow a steady tooth movement by lowering necrosis, and the succeeding hyalinization and indirect resorption, avoiding the recurrent interruptions following blood vessel blockage and reduced additional bone loss. In the present study, the SLBs recorded lower bacterial counts and provide lower force levels, thus allowing more tolerable periodontal reactions. Our results are in accordance with those of Mavreas who reported better periodontal reactions in patients with previous bone loss with SLBs. ${ }^{44}$ Contrastingly; some unexplored variables can have a substantial influence on oral environment. The use of remineralizing agents, fluorides, probiotics, and natural compounds can modify clinical and microbiological parameters in periodontal patients, and they could also have an effect on ALP and IL- 8 levels. ${ }^{45}$ -48 Prospective evaluation of such factors would be imperative to verify their influence during orthodontic treatment procedures.

Limitations of the study include the following: (a) the absence of data after 45 days and at the end of treatment to demonstrate if the periodontal changes occurred would be retained or return to normalcy; and (b) this study was conducted at a single dental center, hence the findings could be generalizable and externally valid, albeit with caution.

\section{Conclusions}

Concerning the materials tested in the present study, the results showed that the conventional brackets conventional brackets ligature technique exhibits greater microbial colonization and higher IL-8 levels and ALP activity compared with the SLB technique. SLBs are therefore more preferable than conventional brackets from the periodontal health perspective. Future clinical trials are recommended to determine the effect of other variables on the clinical, microbial, and chemical parameters over a longer evaluation period.

\section{Abbreviations}

ALP, Alkaline phosphatase; BOP, Bleeding on probing; GCF, Gingival crevicular fluid; GI, Gingival index; IL, Interleukin; PDL, Periodontal Ligament; PI, Plaque index; PPD, Probing pocket depth; SLB, Self-ligating brackets; GPR, Gram positive rods; GPC, Gram positive 
cocci; GNR, Gram negative rods; GNC, Gram negative cocci; GNB, gram negative bacilli.

\section{Data Sharing Statement}

Data are available from corresponding author on reasonable request.

\section{Ethics Approval and Informed Consent}

Written informed consent was obtained from patients or their parents. The protocol was approved from the ethical committee of Imam Abdulrahman Bin Faisal University (2014-01-106). and the study was conducted in accordance with the Declaration of Helsinki.

\section{Acknowledgments}

The authors would like to acknowledge Imam Abdulrahman bin faisal University for funding of this project. We are also thankful for Prof: Khalid Almas and Mr: Faraz Farooqi for their help in unifying the manuscript.

\section{Author Contributions}

Essam Nassar is the main author, All authors made substantial contributions to conception and design, acquisition of data, or analysis and interpretation of data; took part in drafting the article or revising it critically for important intellectual content; agreed to submit to the current journal; gave final approval for the version to be published; and agreed to be accountable for all aspects of the work.

\section{Funding}

This project was supported by a grant from Imam Abdulrahman bin Faisal University but played no role in any part of the study.

\section{Disclosure}

The authors report they have no conflicts of interest in this work.

\section{References}

1. Li Z, Zhou J, Chen S. The effectiveness of locally injected platelet-rich plasma on orthodontic tooth movement acceleration: a systematic review of animal studies. Angle Orthod. 2021;9:391-398. doi:10.2319/061320-544.1

2. Ueda M, Hikida T, Shimizu M, et al. Involvement of interleukins-17 and-34 in exacerbated orthodontic root resorption by jiggling force during rat experimental tooth movement. $J$ World Fed Orthod. 2020;9:47-55. doi:10.1016/j.ejwf.2020.04.002
3. Reiss S, Chouinard MC, Frias Landa D, et al. Biomarkers of orthodontic tooth movement with fixed appliances and vibration appliance therapy: a pilot study. Eur $J$ Orthod. 2020;42(4):378-386. doi:10.1093/ejo/cjaa026

4. Marciniak J, Lossdörfer S, Knaup I, et al. Orthodontic cell stress modifies proinflammatory cytokine expression in human PDL cells and induces immunomodulatory effects via TLR-4 signaling in vitro. Clin Oral Invest. 2019;6:1-9. doi:10.1007/s00784-019-03111-8

5. Nassar EA, Fouda AM, Hassan KS. Influence of low-level laser (LLL) on interleukin 6 (IL-6) levels in gingival crevicular fluid (GCF) during orthodontic tooth movement of periodontally affected rabbits. Int Orthod. 2019;17:227-234. doi:10.1016/j.ortho.201 9.03.004

6. Allgayer S, de Menezes LM, Batista EL Jr. Interleukin 17 (IL-17) and interleukin 23 (IL-23) levels are modulated by compressive orthodontic forces in humans. $J$ World Fed Orthod. 2019;8:148-152. doi:10.1016/j.ejwf.2019.06.001

7. Grieve III WG, Johnson GK, Moore RN, Reinhardt RA, DuBois LM. Prostaglandin E (PGE) and interleukin-1 beta (IL-1 beta) levels in gingival crevicular fluid during human orthodontic tooth movement. Am J Orthod Dentofacial Orthop. 1994;105:369-374. doi:10.1016/ S0889-5406(94)70131-8

8. Kapoor P, Kharbanda OP, Monga N, Miglan R, Kapilla S. Effect of orthodontic forces on cytokine and receptor levels in gingival crevicular fluid: a systematic review. Prog Orthod. 2014;15:1-21. doi:10.1186/s40510-014-0065-6

9. Uitto VL. Gingival crevice fluid-an introduction. Periodontal. 2003;31:9-11. doi:10.1034/j.1600-0757.2003.03101.x

10. Perinetti G, Paolantonio M, D'Attilio M, et al. Alkaline phosphatase activity in gingival crevicular fluid during human orthodontic tooth movement. Am J Orthod Dentofac Orthop. 2002;122:548-556. doi: $10.1067 / \bmod .2002 .126154$

11. King GJ, Keeling SD, Wronski TJ. Histomorphometric study of alveolar bone turnover in orthodontic tooth movement. Bone. 1991;12:401-409. doi:10.1016/8756-3282(91)90029-I

12. Arianda TA, Rezqita P, Pudyani PS, Rosyida NF, Alhasyimi AA. Effect of cocoa administration on osteoblast counts and alkaline phosphatase levels during orthodontic tooth movement in rats. J Orofac Sci. 2020;12:101-106.

13. Yamaguchi M, Shimizu N, Shibata Y, Abico Y. Effects of different magnitudes oftension-force on alkaline phosphatase activity in periodontal ligament cells. J Den Res. 1996;75:889-894. doi:10.1177/ 00220345960750030501

14. Forsberg CM, Brattstro"m V, Malmberg E, Nord CE. Ligature wires and elastomeric rings: two methods of ligation, and their association with microbial colonization of Streptococcus mutans and lactobacilli. Eur J Orthod. 1991;13:416-420. doi:10.1093/ejo/13.5.416

15. Turkkahraman DDS, Sayın DDS, Bozkurt DDS, Yetkin DDS, Kaya S, Onal SL. Archwire ligation techniques, microbial colonization, and periodontal status in orthodontically treated patients. Angle Orthod. 2005;75:227-232. doi:10.1043/0003-3219(2005)075<0227: ALTMCA $>2.0 . \mathrm{CO} ; 2$

16. Prettyman C, Best AM, Lindauer SJ, Tufekei E. Self-ligating vs conventional brackets as perceived by orthodontists. Angle Orthod. 2012;82(6):1060-1066. doi:10.2319/101311-640.1

17. Arteche P, Echandia GO, Sierra A, Aristizàbal JF, Rey D. Considerationes importantes de la ortodoncia con brackets de autoligado versus ligado conventional. Rev Esp Ortod. 2015;45:93-100.

18. Liu Y, Wang PJ, Zhou S, Bai XF. Comparative study of self-ligating orthodontic brackets and conventional brackets: direction and progress. Chin J Tissue Eng Res. 2104;18:4068-4072.

19. Al-Thomali Y, Mohamed RN, Basha S. Torque expression in self-ligating orthodontic brackets and conventionally ligated brackets: a systematic review. J Clin Exp Dent. 2017;9:e123-8. doi:10.4317/ jced.53187 
20. Pandis N, Papaioannou W, Kontou E, Nakou M, Makou M, Eliades T. Salivary Streptococcus mutans levels in patients with conventional and self-ligating brackets. Eur $J$ Orthodontics. 2010;32(1):94-99. doi:10.1093/ejo/cjp033

21. Pejda S, Varga ML, Milosevic SA, et al. Clinical and microbiological parameters in patients with self-ligating and conventional brackets during early phase of treatment. Angle Orthod. 2013;83:133-139. doi:10.2319/010412-8.1

22. Silness J, Loe H. Periodontal disease in pregnancy (II). Correlation between oral hygiene and periodontal conditions. Acta Odont Scand. 1964;24:747-759. doi:10.3109/00016356609028739

23. Löe H, Silness J. Periodontal disease in pregnancy (I) prevalence and severity. Acta Odont Scand. 1963;21:533-551. doi:10.3109/ 00016356309011240

24. Ronald MA. Handbook of Microbiological Media. 2nd ed. New York, Tokyo: Boca Raton; 1997.

25. Deinzer R, Mossanen BS, Herforth A. Methodological considerations in the assessment of gingival crevicular fluid volume. J Clin Perio. 2000;27:481-488. doi:10.1034/j.1600-051x.2000.027007481.x

26. Hassan KS, Alagl AS, Ali I. Periodontal status following self-ligature versus archwire ligation techniques in orthodontically treated patients -Clinical, microbiological and biochemical evaluation. Orthodontic Waves. 2010;69(4):164-170. doi:10.1016/j.odw.2010.05.001

27. Krishnan V, Davidovitch Z. Cellular, molecular, and tissue-level reactions to orthodontic force. Am J Orthod Dentofac Orthop. 2006;129:462-467. doi:10.1016/j.ajodo.2005.10.007

28. Bint Aziz S, Singh G. Cytokine levels in gingival crevicular fluid samples of patients wearing clear aligners. J Oral Biol Craniofac Res. 2020;10:199-202. doi:10.1016/j.jobcr.2020.04.005

29. Başaran G, Özer T, Kaya FA, Hamamci O. Interleukin 2,6, and 8 levels in human gingival sulcus during orthodontic treatment. $\mathrm{Am}$ $J$ Orthod Dentofacial Orthop. 2006;130:e1-7. doi:10.1016/j. ajodo.2005.12.027

30. Gujar AN, Baeshan HA, Alhazmi A, Bhandi S, Raj T, Ptil S. Cytokine levels in gingival crevicular fluid during orthodontic treatment with aligners compared to conventional labial fixed appliances: a 3-week clinical study. Acta Odonto Scand. 2019;77:474-481. doi:10.1080/00016357.2019.1607548

31. Ngan P, Saito S, Lanese R, Shanfeld J, Davidovitch Z. The interactive effects of mechanical stress and interleukin-1 on prostaglandin $\mathrm{E}$ and cycle AMP production in human periodontal ligament fibroblasts in vitro: comparison with cloned osteoblastic cells of mouse. Arch Oral Biol. 1990;35:717-725. doi:10.1016/0003-9969(90)90094-Q

32. Saito S, Sait OM, Ngan P, Lanese R, Shanfeld J, Davidovitch Z. Effect of parathyroid hormone and cytokines on prostaglandin E synthesis and bone resorption by human periodontal ligament fibroblasts. Arch Oral Biol. 1990;35:845-855. doi:10.1016/00039969(90)90010-8

33. Iwasaki LR, Haack JE, Nickel JC, Reinhardt RA, Petro TM. Human interleukin-1b and interleukin-1 receptor antagonist secretion and velocity of tooth movement. Arch Oral Biol. 2001;46:185-189. doi:10.1016/S0003-9969(00)00088-1

34. Perinetti G, Paolantonio M, D’Attilio M, et al. Alkaline phosphatase activity in gingival crevicular fluid during human orthodontic tooth movement. Am J Orthod Dentofacial Orthop. 2002;122:548-556. doi:10.1067/mod.2002.126154
35. Perinetti G, Paolantonio M, Serra E. Longitudinal monitoring of subgingival colonization by Actinobacillus actinomycetemcomitans, and crevicular alkaline phosphatase and aspartate aminotransferase activities around orthodontically treated teeth. $J$ Clin Period. 2004;31:60-67. doi:10.1067/mod.2002.126154

36. Shirozaki MU, da Silva RAB, Romano FL, et al. Clinical, microbiological, and immunological evaluation of patients in corrective orthodontic treatment. Prog Orthod. 2020;21:1-8. doi:10.1186/ s40510-020-00307-7

37. Jingh S, Yuxin L, Jingqiu H, Zhengbin Y, Hui P, Xing C. Comparison of periodontal indices and Porphyromonas gingivalis between conventional and self-ligating brackets. West China J Stomatol. 2013;31:228-231.

38. Sukontapatipark W, El-Agroudi MA, Selliseth NJ, Thunold K, Selvig KA. Bacterial colonization associated with fixed orthodontic appliances. A scanning electron microscopy study. Eur J Orthod. 2001;23:475-484. doi:10.1093/ejo/23.5.475

39. Arbidlo H, Gamarra L, Rojas S, Infantes E, Vàsquez CF. Comparing the periodontal clinical effect between conventional and self-ligating brackets: systematic review and meta-analysis. J Oral Res. 2018;7:155-161.

40. Chhibber A, Agarwal S, Yadav S, Kuo CL, Upadhyay M. Which orthodontic appliance is best for oral hygiene? A randomized clinical trial. Am J Orthod Dentofacial Orthop. 2018;153:175-183. doi:10.1016/j.ajodo.2017.10.009

41. Shin K. Self-ligating brackets may not have clinical advantages over conventional brackets for the periodontal health of adolescent orthodontic patients. J Evid Based Dent Pract. 2017;17:102-104. doi:10.1016/j.jebdp.2017.03.005

42. Arnold S, Koletsi D, Patcas R, Eliades T. The effect of bracket ligation on the periodontal status of adolescents undergoing orthodontic treatment. A systematic review and meta-analysis. J Dent. 2016;54:13-24. doi:10.1016/j.jdent.2016.08.006

43. Nunes L, Quintanilha L, Perinetti G, Junior JC. Effect of orthodontic force on expression levels of ten cytokines in gingival crevicular fluid. Arch Oral Biol. 2017;76:70-75. doi:10.1016/j. archoralbio.2017.01.016

44. Mavreas D. Self-ligation and the periodontally compromised patient: a different perspective. Semin Orthod. 2008;14:36-45. doi:10.1053/j. sodo.2007.12.004

45. Scribante A, Dermenaki Farahani MR, Marino G, et al. Biomimetic effect of nano-hydroxyapatite in demineralized enamel before orthodontic. Bonding of brackets and attachments: visual, adhesion strength, and hardness in in vitro tests. Biomed Res Int. 2020;2020:6747498. doi:10.1155/2020/6747498

46. Rizzardi KF, Rodrigues LKA, Steiner-Oliveira C, Nobre-Dos-Santos M, Parisotto TM. Plaque fluoride levels as a predictor of caries development in early childhood with high sugar exposure a preliminary study. Clin Cosmet Investig Dent. 2020;9(12):71-78. doi:10.2147/CCIDE.S230809

47. Butera A, Gallo S, Maiorani C, et al. Probiotic alternative to chlorhexidine in periodontal therapy: evaluation of clinical and microbiological parameters. Microorganisms. 2020;9(1):69. doi:10.3390/ microorganisms 9010069

48. Costa-Pinto AR, Lemos AL, Tavaria FK, Pintado M. Chitosan and hydroxyapatite based biomaterials to circumvent periprosthetic joint infections. Materials (Basel). 2021;14(4):804. doi:10.3390/ ma14040804 


\section{Publish your work in this journal}

Clinical, Cosmetic and Investigational Dentistry is an international, peer-reviewed, open access, online journal focusing on the latest clinical and experimental research in dentistry with specific emphasis on cosmetic interventions. Innovative developments in dental materials, techniques and devices that improve outcomes and patient satisfaction and preference will be highlighted. The manuscript management system is completely online and includes a very quick and fair peer-review system, which is all easy to use. Visit http://www.dovepress.com/testimonials.php to read real quotes from published authors. 